\title{
La formación epistemológica de profesores de física respecto de la actividad científica
}

Artículo recibido: 02-06-2010 | Artículo aprobado: 02-07-2010

\author{
Physics Teachers' Epistemological Training about \\ Dynamics of Scientific Activity
}

\section{Stellla Maris Islas*}

Margarita Sgro**

Marta Pesa***

Resumen: El artículo presenta algunos resultados de una investigación sobre formación epistemológica de profesores de física en universidades argentinas. Dentro de esta formación se indagaron específicamente los contenidos referentes a la dinámica argumentativa de la comunidad en la cual se elabora el conocimiento científico.

La indagación sobre ambos temas (formación de profesores y dinámica de la comunidad científica) tomó como referente teórico al pragmatismo peirceano. En este escrito se toman en consideración algunas ideas de Peirce relacionadas con dos formas de inferencia: la abducción y la analogía, y su empleo en la comunidad de físicos; también se ilustra la postura de este pensador respecto del carácter comunitario de la construcción de conocimiento.

Luego se consignan los puntos clave de la información obtenida al analizar planes de estudio y programas de contenidos de los profesorados en física, y documentos emanados del Ministerio de Educación para la formación docente. Esta información, además de mostrar la pertinencia de incorporar en la formación de profesores temas relativos a las prácticas científicas, permite inferir la factibilidad de tal incorporación.

Palabras clave: formación epistemológica, profesores de física, Argentina, Peirce, abducción y analogía.

\begin{abstract}
This article presents some outcomes of a research on epistemological Physics teachers' training in Argentinean universities. The authors studied specifically contents concerning argumentative activities in the community where scientific knowledge is constructed.
\end{abstract}

The inquiry on both subjects (Teachers' Training and Scientific Activity) has been based on Peircean Notions of Pragmatism as a theoretical framework. This report takes some Peirce's ideas about two ways of inference: Abduction and Analogy, and their use in Physicists' Community; besides, the position of this thinker according to the social character of knowledge construction.

Finally, the authors include the key points from information obtained by analyzing curriculum and programs for Physics Teachers' Training, and the relevant documents from the Ministry of Education for teachers' training. This information not only show the relevance to include teacher training issues related to scientific practice, but also allow teachers to infer the feasibility of such inclusion.

Keywords: Epistemological Education, Physics Teachers, Argentina, Peirce, Abduction and Analogy.

* Grupo de Actividades Científico-Tecnológicas "Educación en Ciencias con Tecnologías". Facultad de Ciencias Exactas. Universidad Nacional del Centro de la Provincia de Buenos Aires. Campus Universitario. Tandil (7000). Argentina.sislas@exa.unicen.edu.ar

* Facultad de Ciencias Humanas. Universidad Nacional del Centro de la Provincia de Buenos Aires. Argentina. msgro@speedy.com.ar

*** Facultad de Ciencias Exactas y Tecnología. Universidad Nacional de Tucumán. Argentina. mpesa@herrera.unt.edu.ar 


\section{Introducción}

\begin{abstract}
"Un físico descubre algún nuevo fenómeno en su laboratorio. ¿Cómo sabe que las conjunciones de los planetas no tienen algo que ver con él o que no se deba quizás a que coincidió que la emperatriz viuda de China pronunció al mismo tiempo hace un año alguna palabra de poder místico o a que algún genio invisible pueda estar presente? Pensad en cuántos trillones de trillones de hipótesis pueden hacerse, de las que sólo una es verdadera; y después de dos o tres o, como mucho una docena de conjeturas, el físico acierta casi exactamente con la hipótesis correcta. Probablemente no conseguiría eso por azar en todo el tiempo que ha transcurrido desde que la tierra se solidificó" (Peirce, CP 5.172, 1903).
\end{abstract}

Este trabajo está en estrecha relación con una tesis para el Doctorado en Educación, que estudia la formación epistemológica de los profesores de Física respecto de los debates que se dan en la comunidad de investigadores en la disciplina. La relación entre la educación y la cita tomada como epígrafe se comenta en este artículo, planteando el valor que pueden adquirir los saberes epistemológicos de los profesores acerca de los modos de razonar de los científicos.

Vale anticipar que si bien el Pragmatismo de Peirce no se aboca, como tema central, a las cuestiones de la educación formal, algunas de sus ideas, en particular las que caracterizan al aprendizaje como un fenómeno semiótico en el cual tienen un rol decisivo las representaciones construidas por el aprendiz, son ponderadas como fructíferas para mejorar la comprensión de ciertos hechos educativos.

Las líneas de pensamiento peirceano pueden hacer grandes aportes para un estudio pedagógico-didáctico (más adelante aclaramos esta denominación) como el presente, cuando se adopta una mirada epistemológica afín con el Pragmatismo. Para tal fin ha de tenerse la precaución de no homologar los procesos de producción de conocimiento que se dan en la ciencia con los que ocurren en la educación formal, pues se trata de dos contextos epistémicos diferentes.

El enfoque peirceano se considera fructífero, además, porque posibilita incorporar en una tríada semiótica a la dimensión filosófica de la enseñanza de las ciencias. Siguiendo la línea propuesta por Tseitlin y Galili (2007), una herramienta útil para analizar la influencia de la filosofía de la ciencia en la enseñanza de la misma es un triángulo cuyos vértices están constituidos por: ciencia - enseñanza de la ciencia - filosofía de la ciencia, en correspondencia con la tríada propuesta por Peirce: objeto - signo - interpretante. El desarrollo de las significaciones involucradas en estas relaciones excede los límites de este trabajo.

Aquí se ha de considerar la fertilidad de dos nociones peirceanas para estudiar la dinámica de una comunidad de investigadores, dinámica sobre la cual los profesores de ciencias suelen tener escasa información. Tales nociones son:

- la relevancia de la abducción en los debates científicos (a ella alude la cita colocada al comienzo de este artículo) y

- el carácter consensual de la generación de novedades en la ciencia.

Se toman en cuenta también algunas de las actuales tendencias en la interpretación del pensamiento científico, aquellas que consideran al investigador como un individuo que construye modelos representativos 
de las situaciones que estudia (Nersessian, 2006; Giere, 2002), y se vale de ellos para resolver problemas y comunicar resultados a sus pares. La elaboración de un modelo implica la formulación de alguna hipótesis respecto del hecho en estudio (Van Driel y Van Der Valk, 2007). La filosofía de Peirce permite interpretar este proceso, al proponer que la generación de novedades científicas puede ser entendida como un interjuego en el cual intervienen los tres tipos básicos de inferencias (inducción, deducción, abducción) que conducen hacia lo que Magnani (2005) denomina acción creativa del científico, y que conllevan, necesariamente, el intercambio de ideas con los pares.

En este artículo se exponen ejemplos de los modos de inferencia analógicos y abductivos que son usados por científicos en las oportunidades de discusión que les ofrecen las revistas especializadas. Tales ejemplos proceden del estudio realizado en dichas revistas sobre un tema de Física (refracción con índice negativo) que actualmente suscita debates. Se hizo un relevamiento de las ternas argumentativas que se generan a partir de un determinado trabajo publicado; las ternas están conformadas por: la publicación original, otra publicación que la cuestiona, y una tercera, que es la respuesta del autor.

Finalmente, se presentan en forma concisa los resultados obtenidos al analizar los programas de contenidos de los Profesorados que se dictan en universidades argentinas y los lineamientos curriculares propuestos para ellos. Estos resultados permiten afirmar que en la formación de profesores la dinámica de la comunidad de investigadores es poco estudiada, aunque es posible incorporarla a partir de algunos de los temas que figuran en los programas. Se seguiría de este modo con las propuestas ministeriales recientemente formuladas para nuestro país, donde estamos transitando por una etapa de transformaciones en el terreno educativo. El propósito general para esta investigación es, precisamente, el de ofrecer fundamentos de investigación para el diseño de innovaciones en la formación de profesores de Física.

\section{Los estudios sobre la actividad de los científicos}

Las interacciones sociales que se dan en el seno de las comunidades científicas no estuvieron inscriptas en los esquemas de análisis de las epistemologías estándar. La mirada sobre la ciencia como un todo excluía a quienes construyen el conocimiento científico; y posturas como las de Bachelard y Peirce hubieron de esperar muchos años para ser incorporadas a las grandes discusiones de la filosofía de la ciencia.

El falibilibismo iniciado por Peirce y Popper, aunado con programas no positivistas como los de Kuhn, Lakatos y otros epistemólogos no estándar abrieron el espectro para la Filosofía de la ciencia, que estaba "recluida" en el contexto de justificación, en busca de la certeza, que es una aspiración que puede sostenerse "cuando tratamos con abstracciones lógico-lingüísticas, pero no cuando trabajamos en el terreno de la praxis" (Marcos, A. 2008:23). Las investigaciones sobre el proceso de construcción de la ciencia llevan la atención al espacio de actividad de los científicos. Ellos son vistos como sujetos epistémicos diferentes a los que veía la concepción heredada, pues

"la ciencia sigue considerándose como un diálogo, pero no entre un sujeto objetivo y la naturaleza, sino entre sujetos intencionales, comunidades (entre dos individuos, o entre un sujeto y la comunidad), y la naturaleza de fondo" (Santana de la Cruz, 2004). 
Al valorar la importancia de las interacciones dialógicas entre científicos se está justipreciando la dimensión retórica de la ciencia $y$, con ella, una noción de racionalidad que es más amplia que la tradicional, pues coloca a la ciencia en el terreno de la acción.

A partir de mediados del siglo XX toman fuerza creciente las visiones que buscan respuestas a interrogantes sobre el que fuera denominado contexto de descubrimiento. La creatividad puesta en juego en la construcción de la ciencia y las acciones recíprocas que se dan entre quienes realizan esa construcción se erigen como asuntos de interés; se toma en cuenta la semántica de los enunciados, y se reconoce la importancia de la modelización.

Recientemente (a partir de la década del 90 del siglo XX) ganan consenso las investigaciones que muestran al pensamiento analógico como un protagonista importante en el proceso de interpretación de resultados, así como en el de creación de conceptos científicos (Nersessian, 1992). La analogía y la abducción, siendo formas de inferencia que carecen de validez lógica para establecer resultados concluyentes, se reconocen como estrategias potentes para generar hipótesis; la abducción permite modelar el proceso creativo de generación de hipótesis de una manera explícita y formal, retirándolo del terreno de la irracionalidad (Magnani, 2005).

La jerarquía de la abducción como inferencia esencial en la formulación de hipótesis científicas a la cual alude la frase de Peirce colocada al inicio de este artículo, es una categoría reconocida actualmente de modo creciente (Kapitan, 2000; Zeccheto, 2005; entre otros). Suele ser catalogada como su contribución más original a la lógica de las inferencias; en su momento, el propio Peirce hizo explícito su reconocimiento a la for- mulación original de Aristóteles (Marafioti, 2004). Al realizar abducción, el universo de hipótesis susceptibles de ser aplicadas a la resolución de un problema resulta acotado a un número que no sólo es finito, sino que se torna accesible a la contrastación.

\section{La inferencia abductiva}

Esta forma de inferencia está presente con frecuencia en los razonamientos cotidianos y en los científicos (Calvo, 1996; Aliseda, 2006). Sin embargo, es habitual que se le preste poca atención en los cursos y textos que analizan las maneras de razonar; en ellos abundan formalizaciones y ejemplos de inducción y de deducción, pero no de abducciones. Es por este motivo que a continuación se ofrece una breve explicación, que el lector avezado en asuntos lógicos encontrará ya conocida.

La obra de Charles Peirce "rescata" (al decir de Samaja, 1994:82) el valor de la abducción en la tarea de formulación de hipótesis. Un punto básico es que el camino al conocimiento se emprende cuando el sujeto, sea éste un científico o no, se enfrenta con algún hecho que suscita una duda, una pregunta, un problema. Pero esto no es suficiente: "Si no se poseyera alguna creencia de lo que debiera ocurrir de acuerdo con la teoría que se sustenta, ningún acontecimiento podría ser identificado como un fenómeno extraño o problemático" (Calvo, 1996:167). Una vez reconocido el hecho como incomprensible a la luz de lo ya conocido, se intenta formular alguna conjetura, un enunciado hipotético que pueda orientar hacia la explicación de ese hecho, y para ello se apela al acervo de saberes previos.

Mediante esta operación, el hecho pasa a tomar el carácter de indicio. Por ejemplo: 
un antropólogo encuentra restos de ciertas vasijas. Él sabe que esas vasijas eran construidas por determinada comunidad y que los restos de una cultura se hallan, generalmente, en los espacios donde residió la comunidad que los elaboraba. Concluye que el sitio que está recorriendo podría haber estado habitado por esa comunidad.

Un análisis a posteriori del razonamiento muestra que éste se inicia con un enunciado singular, que conduce a evocar una regla general conocida. La conclusión que se obtiene es que el hecho sobre el cual predica el enunciado singular puede ser encuadrado como un "caso" de esa regla.

Para este ejemplo, la regla puede formularse así: "Si una comunidad residió en un lugar, allí se encontrarán restos de los objetos que elaboraban". Es un enunciado del tipo: " $p$ " implica " $q$ ". El antropólogo está en frente a " $q$ " (la presencia de restos) y a partir de esto, mediante la regla general, infiere " $p$ " (aquí residió tal comunidad). Es lo que se da en llamar una "retroducción", pues el pensamiento va hacia atrás de la premisa inicial, recuperando algo previamente conocido.

La sucesión de premisas tiene, en la abducción, una secuencia diferente a la cadena deductiva, dado que ésta toma a la regla general como punto de partida. En la inferencia abductiva la cadena comienza con un evento singular, y la regla general es invocada a partir del mismo.

\section{La inferencia abductiva en los razonamientos científicos}

El ejemplo que se acaba de comentar corresponde a una inferencia típica de los estudios científicos en Antropología. Más adelante en este artículo se presentarán ejemplificaciones provenientes de la inves- tigación en Física. Pero antes de ello, se considera pertinente profundizar en consideraciones atinentes a la actividad científica en sí misma, más allá de las particularidades propias de cada campo de estudio.

Se ha caracterizado ya a la abducción como un "camino desde los hechos hacia las ideas" (Hoffman, 1999), siendo la primera maniobra intelectual a la cual se apela ante fenómenos cuya explicación se desea hallar. Los conocimientos previos de quien razona permiten construir un marco de aceptabilidad para las ideas que surgen en esa búsqueda de explicación y, como consecuencia, se dejan de lado las ideas que son inaceptables y se continúa razonando a partir de las demás. A esta selección de ideas se refiere Peirce en la cita tomada como epígrafe para este artículo, en la cual este pensador (además de caricaturizar un poco con menciones a planetas y a emperatrices) señala que habitualmente son pocas las conjeturas que el científico selecciona para poner a prueba.

La forma de razonar que se adopte para construir conocimiento también dependerá de los hábitos culturales de quien lo hace, pues ellos marcan preferencias hacia determinadas formas:

"Lo que nos lleva a seguir, a partir de unas premisas, una forma de inferencia, es un hábito de la mente, que puede ser constitutivo o adquirido" (Peirce, 1877).

Es decir, sobre la base de lo ya conocido, se razona adoptando caminos que se relacionan con los recorridos anteriormente en otros aprendizajes, que en el caso del científico son los típicos de su disciplina.

Tales caminos distan de ser lineales, pero pese a la variedad de formas que puede adoptar una línea argumental compleja, los tipos de inferencia que componen cada uno 
de sus pasos pueden ser: abducción, inducción y deducción.

"... hay solo tres formas elementales de razonamiento. La primera, a la cual llamo abducción [...] consiste en examinar una masa de hechos y darles la posibilidad de sugerir una teoría. En esta forma obtenemos nuevas ideas, pero no hay fuerza en el razonamiento" (Peirce, CP 8.209).

La falta de "fuerza" de esta forma de razonamiento se debe a que el resultado de una inferencia abductiva -cuyas premisas sean verdaderas- no es una conclusión necesariamente verdadera, sino solamente plausible (su formato lógico corresponde a la falacia de afirmación del consecuente). Se obtiene una hipótesis, un enunciado verosímil que ha de someterse a prueba usando las otras dos formas básicas de inferencia. Una vez que la abducción ha permitido arribar a una hipótesis, pueden generarse condicionales (deductivos) en los cuales tal hipótesis es el antecedente, y el consecuente es una predicción que puede contrastarse (Kapitan, 2000). Se configura así un ciclo entre las tres formas básicas de inferencia, un proceso que, si fuera continuado indefinidamente, resultaría en una "convergencia hacia la verdad" (Marafioti, 2004).

En algunas ocasiones Peirce habla de la abducción relacionándola con un "instinto de adivinación" (CP 7.46). El calificativo de "adivinatorio" alude al carácter espontáneo de la generación de hipótesis; pero cuando se estudia retrospectivamente esta generación, es posible encontrar la lógica subyacente si se tiene en cuenta que

"el conjunto elegido de posibles hipótesis está determinado por un cierto conjunto de contextos que son asumidos como relevantes en una situación histórica determinada (Hoffman, 1997, pág. 7).
El acto de producir hipótesis no está, pues, regido por el azar, sino que depende del contexto, así como de las semiosis previas. Depende de los hábitos instalados previamente en el individuo y que se aplican a situaciones nuevas, conjugando así una operación lógica que es la única capaz de introducir una idea nueva (Peirce, CP 5.171); las otras operaciones lógicas básicas (inducción y deducción) no aportan contenidos semánticos que no estén ya presentes en las premisas. De aquí la importancia de la abducción en la generación de novedades científicas (Campos, 2009).

\section{El uso de analogías en la investigación científica}

Este uso reviste una gran importancia en la tarea de los científicos, así como en la construcción de conocimiento cotidiano. Las conductas creativas no son susceptibles de reducirse a formas algorítmicas de razonar (Nersessian, 2002), ellas suscitan la elaboración de analogías entre dos sistemas, al reconocer similitudes y diferencias entre ellos. Para tal fin, la analogía se basa en ciertos atributos de las situaciones que interesan. Esta clasificación y la consecuente selección de atributos no está inscripta de modo fijo en cada sistema que se estudia, pues puede variar en función del estudio en sí, (Acevedo, 2004) de modo tal que en un caso un atributo puede considerarse como inherente a la esencia del sistema y en otro caso, no.

En general, las inferencias analógicas ofrecen pautas para comprender una situación a partir de la comparación global entre dos sistemas; entre un "todo" y otro "todo" denominados comúnmente dominio fuente y dominio blanco (cconviene recordar que aquí el vocablo "blanco" se toma en el sentido de "objetivo o meta", o bien "destinatario". En Inglés, la palabra es target). 
En el caso de un estudio científico el dominio fuente es un constructo ya validado por la comunidad, y su correspondencia con el dominio que se está indagando forma parte de la propia investigación. Cada uno de estos dominios puede consistir en:

- un hecho natural, o uno producido en laboratorio,

- una interpretación teórica,

- un diseño experimental, su puesta en marcha y su interpretación,

- una modelización,

- una simulación informática.

A veces, el dominio fuente y el dominio blanco son entidades del mismo tipo; ello ocurre en Física cuando se comparan dos experimentos, o dos simulaciones, o dos desarrollos teóricos. Por su parte, y en función de la temática específica que se aborda en cada uno de los dominios, la analogía puede establecerse entre dominios próximos o remotos, y éstos pueden ser entidades del mismo tipo o bien, dominios de diferente naturaleza (Dunbar, 2001).

La elaboración de un modelo científico corresponde al caso de una analogía entre dos dominios ontológicamente diferentes: un hecho natural (sistema real) y una representación del mismo, que puede tomar la forma gráfica, matemática, verbal, o la de un montaje de laboratorio (maqueta). Los modelos cientíícos se conceptualizan como análogos estructurales de los sistemas materiales (Nersessian, 1992). Existe correspondencia entre las propiedades de uno y otro, pero hay propiedades del sistema real que se omiten en el modelo, por ser irrelevantes para el estudio que se está realizando. Esta selección de propieda- des relevantes no es algorítmica, sino que se vale de los significados, con una fuerte carga semántica que puede variar de un individuo a otro, en función de las semiosis que realiza (y ha realizado antes) respecto del tema en estudio, en función también de sus hábitos de pensamiento y, claro está, de los propósitos que persiga su estudio. Por estas razones, la relación sistema realmodelo, no es unívoca, sino que es posible que emerjan diferentes modelos científicos para un mismo hecho.

Algunas controversias entre investigadores se generan porque para un mismo sistema real, califican de modos diferentes a los atributos del mismo; de este modo, un atributo que es relevante en opinión de uno de los investigadores, es considerado despreciable por el otro. Y de allí se sigue que, por ejemplo, difieran las ecuaciones que ellos emplean para representar al sistema real, por estar empleando modelos diferentes.

La creación, el empleo y la evaluación de modelos son tareas esenciales para todo científico. En un trabajo anterior (Islas, 2002. a y b) se documentaron las respuestas de científicos sobre la actividad de modelización. Ellos la señalan como estrategia de abordaje de todo fenómeno, que habilita para trabajar sobre él, sea en forma experimental o como parte de un desarrollo teórico. Este dato empírico concuerda con los de otros investigadores (Adúriz Bravo, 2005; Van Der Valk et al. 2007) y también con estudios más teóricos (ej.: Suárez, 1999) que sostienen que en las bases de las que se valen los científicos para la generación de nuevos saberes, además de los sistemas axiomáticos y las redes proposicionales, tienen relevancia los modelos. Gran parte del entrenamiento de los científicos novatos consiste en aprender los modelos aceptados en su comunidad y desarrollar la capacidad de manipularlos (Nersessian, 1999). 
La historia de la ciencia ofrece ejemplos en los cuales los modelos han precedido a la creación de las leyes y axiomas de las teorías (Nersessian, 2002). Del mismo corte es uno de los registros obtenidos durante el trabajo que hemos realizado con publicaciones científicas: la posibilidad de producir artificialmente materiales a través de los cuales se dé el fenómeno de refracción negativa, que fue anunciada hace unos cuarenta años por un científico soviético, autor del primer modelo de material con esas propiedades, a las cuales el desarrollo científico-tecnológico ha permitido arribar recientemente.

El ejemplo que recién se ha mencionado muestra que algunas ideas imposibles de materializar en determinadas circunstancias (en este caso, por limitaciones tecnológicas) son tomadas luego en otro contexto de desarrollo, y llevadas a la práctica. Puede interpretarse como uno de los casos en los cuales los saberes previos, aunque hipotéticos, valen para nuevos progresos. Al decir de Zamora (2005) la ciencia es una empresa cooperativa en la cual todo proceso de investigación va mucho más allá de lo que un individuo aislado podría emprender; toda investigación parte siempre de un corpus de conocimientos heredados y es precisamente porque existe ese corpus por lo que un investigador puede plantearse resolver un cierto problema. Además de lo heredado, la investigación se vale de los saberes que están circulando en la comunidad a la cual pertenece el científico. A ellas se dedica el apartado siguiente.

\section{Las comunidades epistémicas}

Estas comunidades se caracterizan como grupos de personas ocupadas en la construcción de conocimiento, con una dinámica interna que les es propia y con otra dinámica que involucra su relación con el tejido social en el cual se insertan. Los miembros de una comunidad epistémica comparten intereses, significados, métodos, conceptos.

En los últimos años ha crecido la cantidad y la variedad de estudios sobre estas comunidades, habiéndose agregado intereses que no se presentaban en el pasado: la cientometría, las bases de datos sobre publicaciones científicas disponibles en Internet, responden a necesidades generadas por las nuevas formas de difusión de conocimientos (Roth-Bourgine, 2004), que demandan la apertura a modos no tradicionales de concebir la actividad científica.

Las nuevas tecnologías de la comunicación facilitan la práctica de un hábito que está presente en los científicos desde épocas muy remotas: la interacción con los pares. Puede tomarse a la Modernidad como hito, pues en ese período no solamente se modifican las imágenes de ciencia y de científico, sino que se formaliza la creación las primeras asociaciones de científicos (Reale y Antiseri, 1995).

En nuestros días, las sucesivas mejoras que han venido teniendo los medios de comunicación se potencian con el uso de las herramientas informáticas que, además de expandir el espectro de problemas factibles de abordar por la ciencia, constituyen el medio de mayor eficiencia para distribuir información sobre las actividades que cada científico lleva a cabo en su lugar de trabajo. El flujo comunicativo entre colegas está favorecido; podemos advertir que continúa en vigencia la idea expresada por Peirce hace ya un siglo:

"No llamo ciencia a los estudios solitarios de un hombre aislado. Sólo cuando un grupo de hombres, más o menos en intercomunicación, se ayudan y se estimulan unos a 
otros al comprender un conjunto particular de estudios como ningún extraño podría comprenderlos, [sólo entonces] llamo a su vida ciencia" (Peirce, 1905).

Es natural, para todo científico, enviar sus informes para que sean evaluados por expertos y además sabe que, luego de su publicación, vendrá el juicio de los colegas. Esta es una actitud que se encuadra en el Falibilismo, pues supone aceptar que "por más que uno confíe en la verdad de lo que sabe, siempre puede estar en un error y que esta convicción debe orientar nuestras acciones" (Marcos, 2008:18). La disposición a atender las opiniones de sus pares es un hábito instalado en cada científico, así como la de ofrecer sugerencias que pueden mejorar el trabajo de un colega.

Generalmente los intercambios de ideas surgen cuando los hechos que estudia un investigador son interpretados de manera diferente por uno de sus colegas. Puede decirse entonces que la semiosis que realiza uno de ellos al modelizar el hecho, es diferente de la del otro. Esa diferencia se debe a que ante un mismo signo, es posible que haya más de un interpretante, ya que "el interpretante de un signo refleja siempre los hábitos mentales de la persona que entra en contacto con el representamen" (Zecchetto, 2005:61). La diversidad que poseen los hábitos y conocimientos previos de quienes "leen" los signos promueve a veces la divergencia y las polémicas; en este caso interesan los debates que se dan en el seno de una comunidad de investigadores.

Esta dinámica argumentativa ha sido estudiada por filósofos de la ciencia, existiendo acuerdo en cuanto a que la Historia y la Sociología de la ciencia ponen en claro que es preciso descartar la imagen de la "verdad" científica emanando unívocamente de un estudio, pues "el proceso que lleva a la aceptación de un descubrimiento científico es mucho más tortuoso: siempre hay un cierto grado de flexibilidad en cómo presentar los resultados de investigación, y las interpretaciones finalmente aceptadas son el resultado de una negociación entre varios agentes" (Zamora, 2006:177).

El valor del consenso entre colegas se hace manifiesto en la aceptación de investigaciones a publicar, y a tal punto que la comunicación, además de ser esencial a la naturaleza y práctica de la ciencia, se erige como ámbito de calificación de los científicos:

"El hecho de que el número de publicaciones y artículos se haya utilizado como indicador del crecimiento científico establece la producción de publicaciones del proceso de investigación como medida válida de su actividad" (Russel, 2001).

El prestigio de un investigador, o de un grupo de investigadores se asienta, fundamentalmente, en la difusión que toman sus producciones científicas.

El contenido de los párrafos anteriores es conocido -explícita o implícitamente- por todo investigador, que desde el inicio de su carrera sabe que cada segmento de su trabajo de investigación (desde la revisión de antecedentes hasta el análisis de conclusiones) ha de ser susceptible de tomar un formato escrito que permita ponerlo a consideración de expertos, con miras a publicarlo. La reputación académica, el renombre, y otras formas de reconocimiento profesional toman para el científico un valor mucho mayor que en otras profesiones, siendo el juicio de sus pares la instancia definitoria (Becher, 2001). Y para convencer a sus colegas respecto de la validez de sus hallazgos, es preciso que sepa valerse de argumentos explícitamente justificados (D. Kuhn, 1993), 
además de consideraciones de otros órdenes que le permiten sostenerse en el estilo de trabajo que es propio de su comunidad.

Los nuevos significados que van construyendo los investigadores en su trabajo cotidiano pasan por severos controles en los foros de la comunidad epistémica (Roth y Bourgine, 2005) a la cual el científico pertenece. Esos foros (ya planteados por S. Toulmin en 1977) revisten formas muy diversas, desde las más difíciles de escrutar, que son las discusiones efectuadas en el interior del equipo de investigación (estudiadas por Dunbar, 1999; Nersessian, 2006), hasta las más difundidas, como son las que pueden hallarse en publicaciones especializadas. A un grupo de ellas se ha dirigido el estudio empírico de recoger ejemplos de inferencias analógicas y abductivas, que se comenta a continuación.

\section{Algunos ejemplos de inferencias realizadas por científicos}

Para evitar que este artículo resulte demasiado extenso, se procura conciliar la brevedad del relato con su capacidad para situar al lector en el marco empírico del cual se han extraído los ejemplos.

Algunas aclaraciones sobre el contenido de Física respecto del cual se discute en los artículos analizados: las temáticas novedosas, que suscitan controversias, son el terreno más fértil para localizar discusiones científicas; pero precisamente por versar sobre afirmaciones que aún no han sido consensuadas, emplean conceptos y propiedades poco conocidas. Es éste el caso del tema "refracción de índice negativo", respecto del cual se han analizado discusiones en este trabajo. No es posible realizar en pocas líneas una caracterización rigurosa de este fenómeno. A los fines de este artículo, entendemos que es suficiente con mencionar que este tipo de refracción se produce cuando ciertas radiaciones atraviesan determinados materiales, siendo éstos de difícil fabricación. Resulta de interés para la Física no solamente porque abre posibilidades de mejorar diseños de dispositivos (por ejemplo: antenas) sino también porque implica la revisión de ciertas nociones teóricas.

Quizás sea apropiado reiterar algo ya dicho en la Introducción: se han analizado publicaciones académicas en las cuales se discuten informes de investigación sobre este tema. Las ediciones electrónicas de 18 revistas especializadas han permitido acceder a intercambios de ideas sobre el mismo, en el trienio 2006-2008. El análisis se ha realizado sobre las ternas que conforma un artículo con el comentario que recibe y con la respuesta que publica el autor del artículo original. Se indagaron las formas de inferencia subyacentes a estos escritos científicos, y se consignan aquí ejemplos de inferencias analógicas y abductivas.

\section{Inferencias analógicas}

- Los autores de un comentario cuestionan que para mostrar una de las posibilidades de fabricar materiales en los cuales se dé refracción negativa, se haya empleado la misma ecuación que se utiliza en otro trabajo, diciendo: "Aunque con esto se capturan las contribuciones más importantes, se deja de lado la polarización eléctrica debida a la componente magnética del campo de prueba".

- De este modo, sugieren que la analogía entre el trabajo en cuestión y otro que ya ha ganado consenso ha sido llevada demasiado lejos, puesto que se ha tomado una ecuación en la cual no figura una variable que debería haberse tomado en cuenta, 
por no ser despreciable su influencia en este caso. Algo similar aparece en otro comentario: los comentadores no están de acuerdo con la simplificación que el autor ha efectuado al asumir que determinadas variables toman valores que las convierten en despreciables.

- Una forma más explícita del uso de analogías es la que se presenta cuando los autores de un artículo toman en cuenta que experimentalmente ya se han logrado materiales que provocan refracción negativa mediante arreglos de resonadores (un resonador es un dispositivo material que, al ser sometido a ciertas vibraciones del medio, amplía su propia vibración, es decir, "resuena"; así funcionan las cajas de los instrumentos musicales. Este fenómeno puede darse con ondas mecánicas, como las sonoras, o con ondas electromagnéticas, que es el caso al cual se refiere este ejemplo). En el modelo que los autores proponen, "una transición eléctrica y una transición magnética cumplen el rol de un resonador eléctrico y un resonador magnético, respectivamente".

De esta forma, están señalando la analogía entre un dispositivo material (resonador) y una variación de valores eléctricos y magnéticos.

\section{Inferencias abductivas}

- En uno de los trabajos, los autores muestran teóricamente que en determinado tipo de medios es posible la refracción negativa empleando ondas a frecuencias ópticas (visibles). Los comentadores hacen los cálculos con los valores de parámetros empleados por los autores, y llegan a diferentes conclusiones respecto de la calificación (disipativo/ no disipativo) del medio empleado. Los autores responden que la restricción en valores de variables que los comentadores practican no corresponden al tipo de función que vincula a la longitud de onda con el índice de refracción para ese tipo de medios.

La disidencia proviene de calificar el medio de diferentes maneras. Esta calificación actúa al modo de la "regla general" que en el razonamiento abductivo orienta las decisiones. En este caso, las decisiones atañen a cuál es la ecuación que permite resolver una parte del problema en estudio.

- En otro trabajo, se sustenta la idea de que para tener refracción negativa es imposible eliminar (o reducir casi totalmente) las pérdidas ópticas en toda la región en la cual se da esta refracción, pero que es posible reducirla para algunas frecuencias aisladas; con esto se tendría un criterio para optimizar el diseño de materiales en los cuales este fenómeno se produce. Los comentadores cuestionan las "bajas pérdidas" estudiadas por el autor, proponiendo para esto el análisis de un contra-ejemplo. La respuesta del autor señala que si bien hay concordancia en los planteos teóricos generales, sus colegas no han tenido en cuenta una aclaración que él formula respecto del concepto de "bajas pérdidas" que emplea. Agrega que además, el contra-ejemplo propuesto es posible matemáticamente, pero no físicamente.

La disidencia se produce, nuevamente, por tomar encuadres conceptuales distintos.

- La simulación presentada en otro trabajo muestra que cadenas de ciertas partículas muy pequeñas pueden actuar 
como "nano-lentes" (diminutas lentes). El comentario que se genera no apunta a lo recién afirmado, pues admite que cualitativamente es posible, pero hay disidencia en cuanto a los valores propuestos por los autores. Es desaprobada la propuesta de emplear cierto tipo de aproximación (llamada aproximación cuasi estática) en este caso.

Se está en desacuerdo, entonces, con la modelización efectuada. Los autores insisten, al responder, en la pertinencia de usar su modelo, pues han elegido deliberadamente los parámetros del sistema para que quede dentro de los límites de validez de la aproximación cuasi-estática.

\section{Regresando a la preparación delos docentes}

Las prácticas científicas a las cuales hemos dedicado los apartados anteriores son desarrolladas en universidades donde se forman profesores. Más aún, en muchos casos los futuros profesores cursan asignaturas que están a cargo de investigadores. Empero, como ya hemos mostrado en publicaciones anteriores (Islas, 2009) los estudiantes de profesorado no reciben información sobre estas prácticas por parte de los propios protagonistas de la construcción de conocimiento; hemos dado cuenta (Islas, 2002a) de la exigüidad de los espacios específicos de construcción de saberes sobre la comunidad científica que trabaja en el mismo ámbito institucional en el cual los futuros docentes cursan su carrera.

El camino para la construcción de esos saberes sería el que se transita en las cátedras específicas de la formación docente. A ello apunta nuestro trabajo: sobre la base de los resultados obtenidos en el terreno epis- temológico proponemos algunas innovaciones, fundamentadas en la investigación, para las carreras de formación de profesores, así como para las actividades de formación continua de las que participan docentes en servicio.

Concebimos a estas innovaciones como pedagógico-didácticas, pues con el primer calificativo aludimos al análisis de la compleja constitución del fenómeno educativo que abarca cuestiones histórico-políticas $y$ sociales y que tradicionalmente se ha preocupado por problemas tales como la integración social, la formación de la subjetividad, en fin la reproducción y la innovación en las sociedades complejas. En el campo de las ciencias sociales y de la educación, Pedagogía y Didáctica se separan para su estudio en dos disciplinas diferentes; la primera ha respondido a lo largo de la historia preguntas sobre la legitimidad del acto educativo, su necesidad y su posibilidad; la Didáctica, en cambio, se ha preocupado por responder preguntas que remiten al quehacer de la educación, esencialmente en lo que concierne a contenidos o sea, el qué enseñar y metodologías de enseñanza, es decir, el cómo enseñar. Las ideas del docente respecto de la actividad que genera el conocimiento sobre el cual versan sus clases forman parte de un cuadro amplio que se relaciona con su formación pedagógica; $y$, al mismo tiempo, imprimen un determinado sesgo a sus decisiones didácticas, hecho sobre el cual no entraremos en detalle en este artículo, pero que se encuentra documentado en la literatura especializada (Osborne, 2003).

Nuestros resultados sobre la actividad de los científicos han mostrado que la creatividad requerida para la formulación de nuevas ideas tiene su fundamento en los conocimientos previos de los científicos, que les permiten encontrar reglas que 
incluyan al fenómeno que estudian, así como modelizaciones y/o encuadres teóricos posibles para el mismo.

Por otra parte, al hacer una revisión de los temas que estudian los profesores en su formación, se advierte que estas cuestiones no se toman como relevantes en la mayoría de los programas de contenidos de Epistemología, y ello podría explicar algunos de los problemas que aparecen con las concepciones de los docentes acerca de la ciencia.

Pensando en posibles soluciones, resulta razonable suponer que un profesor que comprendiera el rol de la abducción en el razonamiento científico sería menos proclive a adherir a ciertas distorsiones del concepto de ciencia, tales como: la formulación de hipótesis desprovista de antecedentes, la prioridad del uso de razonamientos inductivos y/o deductivos exenta de componentes semánticos, el carácter dogmático de las aseveraciones científicas.

\section{Escenarios favorables para innovaciones}

Los resultados de nuestra investigación documental sobre las propuestas curriculares son alentadores en cuanto a la factibilidad de innovar la formación de profesores en el sentido que proponemos. Los párrafos que siguen contienen una sucinta caracterización de un trabajo (mayores detalles pueden leerse en Islas, 2010) en el cual analizamos: las directivas emanadas del Ministerio de Educación de la Argentina para los Profesorados en Ciencias; los Planes de estudio de los profesorados en Física en nuestro país $y$, para los profesorados en Física de Universidades estatales, los Programas de contenidos de asignaturas vinculadas con Epistemología.
Por un lado, el análisis de Planes de estudio nos ha mostrado una fuerte presencia de la Epistemología en ellos (en 19 de los 22 Planes analizados). Las epistemologías no standard, cuyo estudio figura como contenido en todos los programas analizados, brindan ocasiones para incursionar en la dinámica interna de la ciencia si se coloca más atención a ciertas nociones desarrolladas en esas líneas de pensamiento, tales como la noción de paradigma en Kuhn, la de programas de investigación en Lakatos. Además, en casi todos los programas se encontró la expresión "Tendencias epistemológicas contemporáneas"; aunque no está seguida de subtítulos que permitan asegurar cuáles se incluyen, habilita a pensar que podrían en un futuro, agregarse aquellas líneas de pensamiento que profundizan en las prácticas de los científicos.

Una adecuada formación de los formadores de docentes podría incentivarlos a extender las temáticas que abordan, a fin de que se amplíe el conjunto de caracteres de la ciencia que estudian sus alumnos. Entre ellos, el carácter socio-argumentativo del proceso de construcción de la ciencia, para cuyo estudio en los Profesorados pueden capitalizarse ideas de las corrientes epistemológicas no standard, susceptibles de ser asociadas luego a las de otros pensadores (tales como Peirce y Bachelard, que no suelen ser encuadrados en esas corrientes).

Las nóminas de contenidos, además de variar en el grado de detalle con el que se presentan, son también variables en cuanto al contenido en sí mismo. Particularmente, cuando se trata de enfoques epistemológicos recientes y de miradas sociológicas al interior de la ciencia. Por tal motivo, puede pensarse que en lo que a contenidos concierne, las propuestas 
de cambio encontrarían variadas facilidades de implementación en las diferentes universidades. Esto es, las modificaciones de contenidos que serían pertinentes significan una innovación en la formación de docentes que, para la mayoría de los programas de contenidos, no requiere de modificaciones sustanciales.

No puede olvidarse, sin embargo, que el agregado de contenidos a los programas no comporta en sí mismo un cambio apreciable. Los conocimientos de Epistemología podrán afectar en más o en menos a las concepciones de los docentes, según cómo actúen los diversos condicionantes del aprendizaje que influyen en la noción de ciencia. Sostenemos que -sin desmedro de la consideración de otros factoresincorporar el debate en las clases donde se forman docentes es una buena vía para enriquecer la construcción de significados acerca del proceso de la ciencia. El dogmatismo que muchos docentes atribuyen a la ciencia podría ser enfrentado mediante la comprensión de sus discusiones internas, la valorización de la duda como estímulo para generar conocimiento y el reconocimiento de que la tarea científica no es una simple cadena lineal de aciertos.

La propuesta de desarrollar las competencias argumentativas de los docentes es afín a los lineamientos que el Ministerio de Educación de la Argentina ha diseñado para la formación de profesores. En estos lineamientos se propone que las clases para docentes adopten un estilo que les estimule a asumir un rol más participativo, más activo. Se recomienda superar el formato escolar que es tradicional en esas clases.

Estas recomendaciones parten de reconocer que los docentes frecuentemente replican los modelos de enseñanza con los cuales fueron formados, es decir, suelen apoyarse más en su experiencia como alumnos que en las propuestas estudiadas en los textos de didáctica. Tomando en cuenta esta situación, las recomendaciones orientan a modificar la dinámica de las clases de formación docente.

En los Documentos ministeriales se hallan también evidencias de un interés por la fundamentación teórica de las innovaciones (nótese que ha sido éste uno de los propósitos de nuestra investigación), y de una preocupación por mejorar las nociones epistemológicas de los profesores de ciencias. Se recomienda orientarlos hacia la valoración del trabajo de los científicos en forma comunitaria para interpretar el mundo, intercambiando ideas a partir de las representaciones que cada uno de ellos genera en esa interpretación. Esta recomendación está en línea con las ideas de Peirce, quien ha señalado que una cualidad esencial del proceder científico es la de ser público y que sus actividades son auto-correctivas.

La concepción peirceana aparece también cuando en estos Documentos se analizan las características de la investigación educativa, valorándose los roles de la analogía y de la abducción en la construcción de conocimiento.

Finalmente, vale destacar que la formación de formadores (que mencionamos en la página anterior) es presentada como uno de los temas que las autoridades educativas propenden a enriquecer. Podemos suponer, entonces, que esto podría expandir el espectro de líneas epistemológicas que estudian los profesores y al mismo tiempo podría animar a quienes los forman a incursionar en prácticas didácticas que hoy día son poco frecuentes, como es el caso de las discusiones en torno a los temas en los cuales se basa la clase. 


\section{Comentarios finales}

Se ha intentado mostrar el modo en que algunas ideas peirceanas ayudan a interpretar ciertos aspectos de la actividad científica. Y especialmente ha interesado la disposición de los científicos para participar en intercambios argumentativos, sea presentando su trabajo a la comunidad de pares o bien aportando ideas para otras investigaciones.

Estas actividades no tendrían lugar si el científico, cada vez que arriba a un resultado, creyera que con toda certeza ha logrado una "verdad"; $y$, aunque no hay razones para creer que los investigadores estén siguiendo la máxima de Peirce: Don't block the way of inquiry (No bloquear el camino de la indagación), se constata que, al menos en los casos estudiados, está en ellos la disposición para admitir errores propios y ajenos, y para actuar en un contexto de dudas genuinas, generadoras de un estado de insatisfacción que estimula la exploración de soluciones a los problemas que la ciencia procura dilucidar.

El contraste entre lo que se acaba de consignar y las nociones de ciencia que circulan en nuestras escuelas puede moderarse si en la formación de profesores se intensifica el estudio de las prácticas científicas, especialmente de su dimensión argumentativa y social.

\section{Referencias bibliográficas}

Acevedo D., A. (2004). El papel de las analogías en la creatividad de los científicos: la teoría del campo electromagnético de Maxwell como caso paradigmático de la historia de las ciencias. Revista Eureka sobre Enseñanza y Divulgación de las Ciencias, 1 (3): 188-205.
Adúriz Bravo, A. (2005). Una introducción a la naturaleza de la ciencia. La epistemología en la enseñanza de las ciencias naturales. Buenos Aires, Argentina: Ed. Fondo de Cultura Económica.

Alisefda, A. (2006). Abductive Reasoning. Logical Investigations into Discovery and Explanation. Dordrecht, The Netherlands: Ed. Springer

Becher, T. (2001). Tribus y territorios académicos. Barcelona, España: Ed. Gedisa.

Dunbar, K. (2001). What Scientific Thinking Reveals About the Nature of Cognition. In: Crowley, K., Schunn, C. D., \& Okada, T. (Eds.) (2001). Designing for science: Implications from everyday, classroom, and professional settings (pp. 115-140). Mahwah, NJ: Lawrence Erlbaum Associates.

Calvo, S. (1996). El pragmatismo y la abducción. En Díaz, E. (ed.) La ciencia y el imaginario social. Pp.166-182. Buenos Aires, Argentina: Editora Biblos.

Campos, D. (2009). On the distinction between Peirce's abduction and Lipton's Inference to the best explanation. Synthese, DOI 10.1007/s11229-009-9709-3.

Dunbar, K (1999). Scientific Creativity. Encyclopedia of Creativity; Vol. 1. Canada: Academic Press MacGill University.

Giere, R. (2002). Distributed Cognition in Epistemic Cultures. Philosophy of Science, 69: 632-644.

Hoffman, M. (1999). Problems with Peirce's Concept of Abduction. Foundations of Science, 4: 271-305.

Islas (2002a). Revista Brasileira de Pesquisa em Educação em Ciência. 2 (2): 102-111 
Islas (2002b). En: Michelini, M. - Cobal, M. (Ed.) Developing formal thinking in Physics (First International Girep Seminar - Selected contributions). Pp. 203-207. Udine, Italia: Ed. Forum, Editrice Universitaria Udinense.

Islas (2009). Revista Ciência y Educação. 15(2): 291-304

Islas (2010). Memorias del X Simposio de Invesigación en Educación en Física. Universidad Nacional de Misiones. Posadas, Argentina: Asociación de Profesores de Física de la Argentina.

Kapitan, T. (2000). Abduction as Practical Inference. Recuperado en Mayo 2010 de: www.digitalpeirce.fee.unicamp.br/kapitan.htm

Kuhn, D. (1993). Science as Argument: implications for teaching and learning scientific thinking. Science Education, 77(3): 319-337.

Magnani, L (2005). An abductive theory of scientific reasoning. Semiotica 153 (1/4): 261-286.

Marafioti, R. (2004). Charles S. Peirce: El éxtasis de los signos. Buenos Aires, Argentina: Ed. Biblos.

Marcos, A. (2008). Filosofía de la Ciencia: nuevas dimensiones. México: Ed. Fondo de Cultura Económica.

Nersessian, N. (1992). How do Scientists Think? Capturing the Dynamic of Conceptual Change in Science. In: Giere, R. (ed.) Cognitive models of Science (pp. 3-45). Minneapolis, USA: University of Minnesota Press.

Nersessian, N. (2002). The cognitive basis of model-based reasoning in science. In: Carruthers, Stich and Siegal (ed.) The cognitive basis of science (pp. 133-156). UK: Cambridge University Press.
Nersessian, N. (2006). The Cognitive-Cultural Systems of the Research Laboratory. Organization Studies, 27(1): 125-145

Osborne, J. (2003). Attitudes towards science: a review of the literature and its implications. International Journal of Science Education, 25 (9): 1049-1079

Peirce, C. S. Collected Papers of Charles Sanders Peirce (1931-1958), 8 volumes, vols. 1-6, eds. Charles Hartshorne and Paul Weiss, vols. 7-8, ed. Arthur W. Burks. Cambridge, Mass.: Harvard University Press

Peirce, C. S. (1877). The Fixation of Belief. Popular Science Monthly, 12: 1-15.

Peirce, C.S. (1905) The Nature of Science. Web del Grupo de Estudios Peirceanos de la Universidad de Navarra. Recuperado en Mayo 2010 de: http://www.unav.es/gep/

Reale, G. y ANTISERI, D. (1995) Historia del pensamiento filosófico y científico. Barcelona, España: Ed. Herder.

Roth, C. y Bourgine, P. (2004). Epistemic communities: description and hierarchic categorization. Mathematical Population Studies, 12(2):107-130.

Russle, J. (2001). La comunicación científica a comienzos del siglo XXI. Revista internacional de ciencias sociales. No dedicado a: $L a$ ciencia y sus culturas. Número 168. Disponible en http://www.oei.es/salactsi

Samaja, J. (1994). Epistemología y Metodología. Buenos Aires, Argentina: Eudeba.

Santana De La Cruz, M. (2004) Controversias científicas y retórica de la ciencia. Actas del "IV Congreso de la Sociedad de Lógica, Metodología y Filosofía de la Ciencia en España". Valladolid, España. 
Suárez, M. (1999). Theories, Models and Representations. In Model-Based Reasoning in Scientific Discovery, L. Magnani, N. Nersessian and P. Thagard (ed.), pp. 75-83. New York: Ed. Plenum.

Tseitlin, M. y Galili, I. (2007). Science Teaching: What does it Means? Science \& Education, 15 (5): 393-417.

Van Der Valk, T., Van Driel, J. y De Vos, W. (2007). Common Characteristics of Models in Present-day Scientific Practice. Research in Science Education, 37: 469-488.

Van Driel, J. y Van Der Valk, E. (2007). Towards a Validated Conception of Scientific Model. In Pintó and. Couso (eds.), Contributions from Science Education Research, 321-332.
Zamora B., J. (2005). "Sociología de la Ciencia y Economía de la Ciencia: otra extraña pareja". Capítulo VI de: Cuestión de protocolo. Ensayos de metodología de la ciencia. Madrid, España: Ed. Tecnos.

Zamora B., J. (2006). Rethoric, Induction and the Free-Speech Dilemma. Philosopy of Science, 73 (2): 175-195.

Zechetto, V. (2005). Seis semiólogos en busca del lector. Buenos Aires, Argentina: Ed. La Crujía. 